\title{
Nuevas tendencias en políticas de garantía de calidad en la educación superior
}

\section{Juan Francisco Perellon}

Département de la Formation et de la Jeunesse

Direction Générale de l'Enseignement Supérieur

Affaires universitaires. Rue de la Barre 8. 1014 Lausanne (Suisse)

Juan-Francisco.Perellon@dfj.vd.ch

\section{Resumen}

La Europa de la educación superior atraviesa transformaciones de una magnitud desconocida hasta ahora. Por todas partes, se trabaja intensamente en la construcción de lo que constituye el objetivo principal de las reformas actuales: el espacio europeo de la educación superior (EEES). La construcción del EEES es una etapa decisiva en el proceso de comercialización de la educación superior. En este marco, la cuestión de la calidad ocupa una posición privilegiada, puesto que se sitúa en la intersección misma de las transformaciones actuales. Crucial en este aspecto, la emergencia de la acreditación como objetivo prioritario de las políticas nacionales de garantía de la calidad marca un momento decisivo de este proceso.

El presente artículo expone la evolución general de los sistemas de educación superior y pone de manifiesto el rol cada vez más importante de las actividades relacionadas con la garantía de la calidad, intentando subrayar ciertos riesgos y derivas observables. Propone una reflexión entorno a tres nociones centrales dentro de los debates sobre la transformación de la educación superior: la calidad, la relación con el mercado y el uso de la información que se puede obtener a través de mecanismos de control de la calidad de los establecimientos de educación superior. Una de las ideas fuertes que intentamos poner en evidencia es el hecho de que, en cuestiones relacionadas con la calidad, las preocupaciones formativas han derivado hacia preocupaciones de carácter mucho más sumativo.

Palabras clave: calidad de la educación superior, acreditación, convergencia europea, comercialización.

\section{Abstract. New Trends in Quality Assurance in Higher Education}

Higher education in Europe is undergoing a period of major transformations. Everywhere, emphasis is being placed on achieving the most crucial objectives of all : the construction of a common European Higher Education Area (EHEA). The EHEA constitutes a decisive step in the commercialization of higher education. In this context, quality assurance has a central role to play, being as it is at the intersection of current transformations. More in particular, the emergence of accreditation as the dominant objective of national policies for quality assurance in higher education can be understood as a crucial moment in the process of commercialization.

The paper underlines this evolution by discussing recent evolutions in the domain quality assurance in higher education and stressing a number of potential risks and nega- 
tive prospects. It offers a reflection organized around three main poles: quality assurance in higher education; the relationships with the process of commercialization and the actual use of the information that be collected through the many existing instruments for quality assurance. The paper strongly argues that a decisive shift has taken place since the late1990 s that as replace formative purposes of quality assurance with summative ones.

Key words: quality in higher education, accreditation, european higher education area, commercialisation.

\section{Sumario}

Introducción La acreditación como objetivo dominante de las políticas de calidad

Del esencialismo al realismo: la «calidad» como problema político Nuevas tendencias en el rol de los estudiantes

El nuevo contexto de las políticas de garantía de la calidad en la educación superior

Elementos de conclusión

Bibliografía

\section{Introducción}

La Europa de la educación superior atraviesa transformaciones de una magnitud desconocida hasta ahora. Por todas partes, se trabaja intensamente en la construcción de lo que constituye el objetivo principal de las reformas actuales: el espacio europeo de la educación superior (EEES). En un contexto más internacional que nunca, caracterizado por la promoción de la movilidad de los estudiantes y del profesorado, por el reconocimiento de los títulos y de los diplomas y su comparabilidad, se presenta como necesaria una mayor armonización de las políticas nacionales de educación superior. Pieza clave en este proceso, la Declaración de Bolonia del 19 de junio de 1999 ocupa hoy el centro de todos los debates. Sus objetivos son ambiciosos. Se pueden resumir en tres orientaciones principales: transparencia, movilidad y calidad. Sobre este último tema, la Declaración subraya la intención de promover la cooperación europea en temas de evaluación, con el objetivo de elaborar criterios y metodologías comparables. Más adelante, tendremos ocasión de discutir este elemento de forma más detallada, así como sobre su integración en las tendencias actuales, pero, por el momento, reconozcamos simplemente la gran generalidad que una tal propuesta conlleva. Sin duda, esta generalidad traduce, por un lado, el difícil equilibrio que hay que encontrar entre las prerrogativas nacionales en materia de educación superior y las exigencias supranacionales de coordinación; mientras que, por el otro, pone en evidencia el hecho de que las experiencias nacionales desarrolladas durante las décadas de $1980 \mathrm{y}$ 1990 han alcanzado sus límites y deben ser modificadas (Haug, 2003). Pero también señalan un elemento crucial dentro de las evoluciones actuales en temas relacionados con la educación superior a nivel internacional: la cada vez 
mayor importancia que se otorga a las consideraciones del mercado, en particular en el marco de las negociaciones sobre el acuerdo general sobre el comercio de los servicios (AGCS) (Knight, 2002; Van Damme, 2002; Westerheijden, 2003 b). En este sentido, se puede avanzar que la construcción del EEES es una etapa significativa en el proceso de comercialización de la educación superior; y, en este contexto, la cuestión de la calidad ocupa una posición privilegiada, puesto que se sitúa en la intersección misma de las transformaciones actuales. Crucial en este aspecto, la emergencia de la acreditación como objetivo prioritario de las políticas nacionales de garantía de la calidad marca un momento decisivo de este proceso. El presente artículo expone la evolución general de los sistemas de educación superior y pone de manifiesto el rol cada vez más importante de las actividades relacionadas con la garantía de la calidad, intentando subrayar ciertos riesgos y derivas observables. Propone una reflexión entorno a tres nociones centrales dentro de los debates sobre la transformación de la educación superior: la calidad, la relación con el mercado y el uso de la información que se puede obtener a través de mecanismos de control de la calidad de los establecimientos de educación superior. Una de las ideas fuertes que intentamos poner en evidencia es el hecho de que, en cuestiones relacionadas con la calidad, las preocupaciones formativas han derivado hacia preocupaciones de carácter mucho más sumativo.

La construcción del EEES va a suponer tener que reunir poblaciones estudiantiles con características extremadamente diversas (Kivinen y Nurmi, 2003), aunque con algo común a todas ellas: el deseo de poder optar al reconocimiento de los diplomas y títulos académicos obtenidos en sus respectivos países. En este sentido, un elemento importante que hay que poner en evidencia es la íntima relación que, según nosotros, existe entre la introducción del objetivo de reconocimiento de los diplomas nacionales y la construcción de un "mercado» de la educación superior en Europa, dentro del cual los diferentes actores pueden disponer de información suficiente como para tomar decisiones racionales en cuanto al «mejor» lugar para realizar sus estudios. Esta evolución marca un cambio importante en la percepción del rol del estudiante dentro de los procesos que persiguen la garantía y/o la mejora de la calidad de la educación superior. De una situación donde el estudiante se constituye como actor en situación de aprendizaje, se pasa a una situación donde el estudiante se pone en una situación de cliente-comprador de un servicio - la educación superior-, del cual debe poder obtener un rendimiento máximo por su inversión. Esta situación no es intrínsecamente propia de la temática relativa a la calidad. Más bien refleja una evolución hacia lo que algunos autores han llamado la «comercialización» de la educación superior. Esta perspectiva nos lleva a investigar las características propias del nuevo "paradigma» dentro del cual se construyen e implementan las políticas relacionadas con la garantía de la calidad en la educación superior, observando también cómo participan de las transformaciones generales de las políticas de educación superior, en particular la(s) forma(s) en que se relacionan con la cuestión de la comercialización de la educación superior. Para ello, empezamos ofreciendo un breve background sobre la emergencia de la cali- 
dad como problema político y sus consecuencias sobre los objetivos perseguidos, luego, presentamos las transformaciones más importantes en el sector de la educación superior en el contexto europeo. En una tercera sección, ponemos en relación la cuestión de la calidad con estas transformaciones antes de discutir el rol de los estudiantes dentro de este proceso.

\section{Del esencialismo al realismo: la «calidad» como problema político}

De entrada, cabe recordar que no existe un concepto genérico de la noción de calidad. Al contrario, la idea de calidad tiene significados múltiples y potencialmente contradictorios según los actores involucrados en los procesos políticos de su definición (Tam, 2001). En realidad, la naturaleza relativa de la noción de calidad en la educación en general y en la educación superior en particular es algo sobre lo cual varios autores han coincidido (Muñoz-Repiso, 1996; de Miguel y otros, 1994). Barnett (1992), por ejemplo, indica que la definición del concepto de "calidad» dentro del marco universitario es, ante todo, el resultado de las posiciones adoptadas por los diferentes actores relacionados, de una forma u otra, con la educación superior. En consecuencia, es imposible pasar por alto las interrelaciones sociales que existen entre estos diferentes actores. Nuestra visión de lo que debe ser la educación superior tendrá una influencia directa sobre nuestra manera de pensar la "calidad», la forma de conseguirla, de mejorarla y, de forma general, de garantizarla en el marco de procesos específicos. La calidad, finalmente, es un concepto multidimensional y subjetivo, y siempre relativo: relativo según quien lo define, el momento en que lo hace y el espacio geográfico donde se encuentra. Hay tantas definiciones posibles de la noción de calidad como actores implicados multiplicados por los intereses de cada uno de ellos (Brennan y otros, 1992).

La dificultad, por no decir imposibilidad, de llegar a un acuerdo formal sobre la definición del concepto de calidad ha llevado a un desplazamiento del foco de los debates académicos (por ejemplo: Ball, 1985; Bowden y Marton, 1998; Harvey y Green, 1993; Green, 1993). Sin desaparecer por completo, los debates esencialistas, centrados en entender "por dentro» la noción de calidad, han dejado progresivamente paso a otros más directamente relacionados con su utilidad y, de forma más general, a los actores que participan en su definición y realización. Todos ellos tienen un interés propio en que la formación ofrecida sea de cierta calidad, todos ellos tienen una definición propia de lo que debe ser, pero esta definición no es común a todos los actores (Vroeijenstijn 1992). Middlehurst (1992) sigue la misma línea de pensamiento cuando adelanta la cuestión crucial de saber cual es el lugar más legítimo para definir la calidad dentro de la educación superior. En este sentido, la construcción de políticas públicas específicamente orientadas al área de la calidad ha sido un proceso común en la mayoría de países, no solamente de Europa, sino de la mayor parte del mundo.

Abordar la cuestión de la garantía de la calidad como política pública permite no sólo salir de los debates esencialistas sobre la calidad, centrados en la 
búsqueda de una definición —a menudo inútiles pues una tal definición es siempre relacional y subjetiva-, sino también, y de forma muy significativa para nuestro propósito, poner de manifiesto las relaciones de poder que pueden existir entre los diferentes actores implicados en la definición e implementación de las políticas de garantía de la calidad, las relaciones que mantienen sobre los objetivos que deben ser perseguidos por estas políticas.

Sin embargo, asegurar la calidad de las actividades académicas no es una preocupación nueva dentro del marco universitario. Al contrario, la calidad ha centrado las preocupaciones de las universidades desde sus inicios. Al mismo tiempo, sin embargo, estas preocupaciones han quedado tradicionalmente circunscritas al mundo de la educación superior y de sus representantes — principalmente profesores-, con poca, por no decir nula, intervención por parte de actores externos, como autoridades políticas o representantes de la sociedad - mercado del empleo, estudiantes, etc. Uno puede entonces hablar de la calidad como un "no-problema». Su definición, sin ser explicitada, era aceptada y, en cierta medida, comprendida por la comunidad universitaria. Es en este sentido que Polanyi (1966) se refiere a la calidad como un conocimiento tácito.

Esta situación caracterizada por un casi inexistente interés externo sobre temáticas relacionadas con la calidad ha cambiado de forma radical a partir de la década de 1980. Es en este sentido, que es posible hablar de la emergencia de la calidad como problema político. De ser considerada casi como algo «de hecho" y sin preocupaciones mayores, la calidad ha adquirido progresivamente un lugar central en las políticas nacionales de educación superior. Los factores que permiten comprender tal evolución son múltiples y, naturalmente, varían de un contexto nacional a otro. De forma general, sin embargo, se pueden resumir en una combinación de elementos: unos propios del campo de la educación superior y otros derivados de tendencias más generales que, sin ser directamente el producto de este campo, han ejercido una influencia importante.

En lo que se refiere a los primeros, la expansión de los sistemas de educación superior ha sido un elemento clave. En el ámbito europeo, el debate sobre la expansión de la educación superior emergió a finales de la década de 1950 y principio de la de 1960 (Teichler, 1988). Los gobiernos de entonces empezaron a cuestionar la estructura elitista de la educación universitaria e intentaron encontrar soluciones para reducirla. Común a la mayor parte de los países occidentales, si bien con diferencias en la amplitud y en el ritmo de su introducción, este proceso se ha caracterizado por un aumento tanto cuantitativo como cualitativo de las poblaciones estudiantiles (Altbach, 1999; Scott, 1995; Trow, 1974). La expansión ha sido problemática en la medida que ha puesto de manifiesto la necesidad de modificar las estructuras destinadas a sistemas elitistas, donde sólo una minoría de la población seguía estudios universitarios. A pesar de los cambios sociográficos debidos a la nueva composición de las poblaciones estudiantiles, la cuestión de la expansión de la educación superior no hubiera ocasionado demasiados problemas relativos a la calidad de la formación si no hubiera sido por su combinación con la reducción de las ayudas 
económicas a las universidades. La disminución de los presupuestos destinados a la educación superior, comunes a un gran número de países durante las décadas de 1980 y 1990, han puesto de manifiesto el peligro de ver empeorar las condiciones de enseñanza, especialmente en un contexto caracterizado por una necesidad cada vez mayor de hacer frente a una demanda creciente por parte de los estudiantes.

La combinación entre el aumento del número de estudiantes y la concomitante reducción del soporte financiero ha sido clave en la emergencia de la calidad como "problema», como temática sobre la cual las autoridades políticas pueden y deben debatir y, de forma general, afrontar a través de medidas específicas, las políticas relativas a la garantía de la calidad. Esta tendencia ha sido reforzada por un tercer factor, característico de las décadas de 1980 y 1990: el énfasis sobre la necesidad que las universidades puedan "rendir cuentas» de sus actividades y del uso de los medios financieros de que disponen (Crowther-Hunter, 1983; Dunn, 2003; Kogan, 1986; Neave, 1984; Neave y Van Vught, 1991).

\section{El nuevo contexto de las políticas de garantía de la calidad en la educación superior}

Para poder entender de forma completa las transformaciones actuales de las políticas de calidad, resulta necesario ponerlas en relación, por una parte, con el contexto económico internacional — marcado por una mayor competencia entre Europa y Estados Unidos- y, por otra parte, con el ámbito más amplio de las políticas de educación superior propiamente dichas. En este sentido, el contexto actual de la educación superior en Europa está marcado por cambios importantes que, si bien representan una continuación de decisiones y de procesos adoptados en el pasado - los programas de intercambio ERASMUS, SÓCRATES, etc.- , son sin embargo de naturaleza diferente y actúan como aceleradores decisivos de reformas de amplia magnitud en los varios espacios nacionales (Neave, 2003; Hackl, 2001; Van der Wende, 2000). Para nosotros, una de las características nuevas de los eventos actuales reside en la congruencia de tendencias convergentes hacia una mayor y decisiva comercialización de la educación superior.

El elemento clave de estas tendencias se concentra en la construcción del espacio europeo de educación superior (EEES), que debe servir no solamente para la promoción de la movilidad estudiantil a través de la armonización de las estructuras educativas nacionales, sino también para reposicionar Europa dentro del nuevo sistema económico mundial.

Por ello, el EEES se constituye como un medio para la construcción de Europa como espacio económico de primer orden, con la capacidad suficiente como para hacer frente a EEUU o Japón (CoEC, 2003). No cabe aquí discutir la cuestión del desarrollo económico del espacio europeo más allá de las relaciones que este desarrollo puede tener con el sistema de educación superior y su organización. 
Para ello, la construcción del EEES debe pensarse y analizarse conjuntamente con las numerosas iniciativas correspondientes a proyectos desarrollados en el campo de la investigación y el desarrollo (R\&D), especialmente la Declaración de Lisboa de marzo de 2000. En aquella ocasión, el consejo ejecutivo de la Unión puso de relieve su intención de hacer que Europa «se convierta en la economía basada en el conocimiento más competitiva y dinámica del mundo, capaz de crecer económicamente de manera sostenible con más y mejores empleos y con mayor cohesión social».

Sin entrar a valorar la relevancia de un tal objetivo, es importante para nuestro propósito subrayar la relación que existe entre una tal declaración de principios y los instrumentos políticos propuestos para alcanzarlos. En el campo de la investigación y de la educación superior, la creación del espacio europeo de investigación (ERA - European Research Area) y del espacio europeo de educación superior se presentan como elementos básicos para reducir los obstáculos identificados por la Comisión para poder hacer frente a los requisitos de la competencia internacional. Entre los «obstáculos» identificados por la Comisión europea figuran las barreras que limitan aún demasiado la movilidad de los estudiantes y de los investigadores (EC, 2003). Sin pretender ser polémico, estas «barreras» pueden entenderse como los espacios nacionales que, en las áreas de la investigación y la educación superior, influyen de forma determinante en los modos de organización. Es, precisamente, en este ámbito que se deben entender los esfuerzos realizados actualmente.

En el campo de la educación superior, estos esfuerzos se conjugan con el incremento del comercio global de los servicios educativos, principalmente a través del Acuerdo General sobre el Comercio de los Servicios (AGCS). En Europa, existe una combinación entre, por un lado, el proceso de Bolonia, basado en la cooperación transnacional, y, por otro, los acuerdos AGCS caracterizados a su vez por la competencia entre las naciones. En el propósito del presente artículo, se trata más precisamente de la competencia para atraer a estudiantes de otras zonas geográficas, especialmente, en el caso de Europa, de Asia. Y en este caso, como señalaremos más adelante, la cuestión de la calidad de la enseñanza ocupa un lugar muy importante.

Sin embargo, hoy en día, se sabe aún poco sobre los posibles efectos de una adopción total de los AGCS. Lo que sí se puede notar es una combinación entre atracción y cierta aprehensión. Son muchas las universidades que temen que los AGCS puedan limitar las competencias nacionales sobre temas relativos a políticas de educación superior, en particular la posibilidad de forzar una limitación de los subsidios públicos hacia ciertos establecimientos universitarios. Tanto la Asociación de las Universidades Europeas (EUA - European University Asociation) como la Unión Nacional de los Estudiantes Europeos han tomado posiciones claras en contra de los AGCS. La Asociación de Universidades Europeas, por ejemplo, pone de manifiesto el carácter profundamente público de la educación superior europea. Este carácter no debe ser menospreciado, según la Asociación, por los cambios actuales, sino reforzado. Este aspecto más crítico hacia los AGCS se puede apreciar también en el hecho 
que la Comisión Europea decidió no integrar la educación dentro de las negociaciones de los AGCS y limitar sus esfuerzos en los elementos ya puestos sobre la mesa, a saber, los servicios de educación de carácter íntegramente privado, y solamente éstos.

Las discusiones alrededor de los AGCS tienden, sin lugar a duda, a reforzar la dimensión comercial de la educación superior. Este proceso no es, evidentemente, algo nuevo. Durante los últimos veinte años, la movilidad de los estudiantes de la OCDE se ha multiplicado por dos y, siempre en la OCDE, las exportaciones de servicios educativos se suman casi a las de los servicios económicos. La carrera para atraer a los mejores estudiantes y reforzar así la posición de Europa en la sociedad del conocimiento se está acelerando. Prueba - o consecuencia - de ello es la creación del Espacio Europeo de Investigación y del Espacio Europeo de la Educación Superior. En este baile, los EEUU parten con ventaja. Tradicionalmente, se han caracterizado por su fuerte atractivo, pues más de 600.000 estudiantes extranjeros están actualmente inscritos en alguna de sus universidades. Los acontecimientos del 11-S, sin embargo, han puesto un cierto freno a la inmigración de estudiantes, de lo que puede beneficiarse Europa. De particular interés en el ámbito internacional es el mercado asiático, donde encontramos un ejemplo emblemático de la comercialización de la education superior: los salones o «ferias» de la educación, donde universidades del mundo entero invierten grandes cantidades económicas para presentarse a los mejores estudiantes y atraerlos a sus aulas.

Atraer a estos estudiantes implica disponer de sistemas de educación superior atractivos y «comprensibles» desde fuera del ámbito europeo. Por ello, los esfuerzos en materia de cooperación en el campo de la educación superior son, hoy más que nunca, fundamentales.

A pesar de que la cooperación en temas de calidad en el ámbito europeo empezó durante la década de 1990 (CEC, 1995; Thune y Staropoli, 1997), es, sin lugar a dudas, la adopción de la Declaración de Bolonia en junio de 1999, lo que significó un momento central en la construcción de la Europa de las universidades. Esta declaración, firmada por los representantes de veintinueve países europeos, se posicionaba poco sobre el rol de la calidad dentro del proceso general de armonización de la enseñanza superior en Europa. En este sentido, la declaración establecía únicamente el objetivo de desarrollar una dimensión europea en prácticas relacionadas con la garantía de la calidad, con criterios y metodologías similares (Bologna Declaration, 1999), sin dar ninguna orientación práctica en lo que se refiere a la forma de conseguir tal objetivo.

Dos años más tarde, en junio de 2001, los ministros se reunieron de nuevo en Praga para reafirmar las decisiones tomadas en la ciudad italiana. Esta vez, la referencia a la cuestión de la calidad y de su garantía fue más substancial. En un párrafo destinado a la promoción de la cooperación europea en la garantía de calidad, "Los Ministros reconocieron el papel vital que juegan los sistemas que garantizan la calidad en asegurar los estándares de la alta calidad y en facilitar la comparabilidad de las calificaciones en toda Europa. Ellos también 
fomentaron una cooperación más cercana entre redes que aseguren la calidad y el reconocimiento. Hicieron hincapié en la necesidad de una cercana cooperación europea y una mutua confianza en ella y la aceptación de sistemas que aseguren la calidad nacional. Además, animaron a las universidades y a otras instituciones de educación superior a difundir ejemplos de la mejor práctica y a diseñar escenarios para una aceptación mutua de mecanismos de evaluación y acreditación/certificación. Los ministros apelaron a las universidades y a otras instituciones de educación superior, a agencias estatales y a la Red Europea de Garantía de Calidad en la Educación Superior (ENQA), en cooperación con los cuerpos correspondientes de otros países los cuales no son miembros de ENQA, a colaborar en el establecimiento de un marco de trabajo común de referencia y a difundir la mejor práctica» (Prague Communiqué, 2001).

En Berlín, en septiembre del 2003, se avanzó hacia una etapa sucesiva. En este contexto, los ministros responsables de la educación en Europa dieron un paso más hacia el reconocimiento de la cuestión de la calidad como uno de los elementos fundamentales dentro de la construcción del EEES. Por ello, se comprometieron a desarrollar la acreditación de calidad tanto en el ámbito institucional como en el nacional y el europeo. En este sentido, uno observa cómo, de un proceso marcado principalmente por interrogantes nacionales, se pasa progresivamente a un referente de acreditación, como respuesta a la variedad nacional en materia de garantía de la calidad de la enseñanza superior. Más importante es el énfasis puesto en la necesidad de perseguir la colaboración con el fin de desarrollar "criterios mutuamente compartidos y metodologías para acreditar la calidad", pero siempre con la institución como último actor responsable de la garantía de la calidad de la enseñanza que dispensa. De forma sorprendente, si se compara con las experiencias anteriores, los ministros propusieron una agenda que cada país debería realizar para el año 2005, fecha en la cual se reunirán de nuevo en Bergen (Noruega). Los objetivos son: a) la definición de las responsabilidades de cada órgano, agencia, instituciones u otros actores involucrados en el proceso de acreditación; b) realizar evaluaciones de programas o instituciones, incluyendo la valoración interna, la revisión externa, la participación de los estudiantes y la publicación de los resultados; c) la presencia de un sistema de acreditación, certificación o procedimientos comparables, y d) la participación internacional, la cooperación y las redes de trabajo (Berlin Communiqué, 2003). Un elemento importante para la realización efectiva de estos objetivos es la confirmación del rol que otorgan los ministros de educación europeos a las estructuras de cooperación existentes en el campo de la garantía de la calidad, especialmente la European Network for Quality Assurance (ENQA).

Desde la firma de la Declaración de Bolonia hasta la publicación del Comunicado de Berlín, han transcurrido cuatro años durante los cuales el panorama de la educación superior europea han cambiado de manera radical y durable. En el corazón mismo de estas transformaciones, la cuestión de la calidad ha pasado de ser una mera «dimensión» — como indicaba la Declaración 
de Bolonia- a ser un elemento estratégico fundamental en la construcción del EEES.

Esta transición marca el reconocimiento del cambio de naturaleza de dos elementos que nos parecen fundamentales. Por una parte, encontramos una reformulación de la noción misma de calidad y, más allá, de los objetivos políticos que persigue. En este sentido, se puede observar un desplazamiento evidente de consideraciones formativas hacia consideraciones mucho más sumativas que consagran la acreditación como objetivo prioritario de las políticas nacionales de garantía de la calidad en la educación superior (Haakstad, 2001; Westerheijden, 2003a; Westerheijden y Van der Wende, 2001). Por otra parte, pero de forma paralela, también se modifica el rol de los estudiantes dentro del nuevo marco de la educación superior tal y como se dibuja en el contexto europeo actual. Veamos un poco más atentamente cómo se conjugan estos dos elementos.

\section{La acreditación como objetivo dominante de las políticas de calidad}

Dentro del marco de la Declaración de Bolonia, la cuestión de la acreditación ha adquirido una doble característica. Por una parte, la acreditación se inscribe dentro del proceso de redefinición de las relaciones entre autoridades políticas y universidades, caracterizada por el cambio de los mecanismos de pilotaje de las universidades basados sobre los inputs, a mecanismos basados sobre los outputs, es decir, sobre lo que éstas producen en términos de estudiantes, de resultados de investigación, etc. Esta redefinición se encuentra reforzada por la importancia de la internacionalización de la educación superior, caracterizada por una mayor movilidad de los estudiantes, a la cual se añade la competencia entre las universidades para atraer a los mejores estudiantes. Por otra parte, la emergencia de la acreditación en el ámbito europeo se desarrolla en paralelo a una tendencia fuerte hacia la armonización de los sistemas nacionales de educación superior - a través de la refundación de los programas de estudio-y, como consecuencia, de las políticas de garantía de la calidad. En este sentido, la Comisión Europea recuerda la necesidad de centrar los esfuerzos en el desarrollo de criterios comunes de calidad a nivel europeo, con el fin de asegurar su transparencia, comparabilidad y compatibilidad (Martens y Balzer, 2004; Zgaga, 2003). Así pues, más allá de una cuestión de comparación formal de la estructura de los programas de estudios - la conocida trilogía Bachelor-Master-Ph.D. común a los países signatarios de la Declaración de Bolonia-, la construcción del EEES se presenta como un proyecto doble que nos permite entender por qué la acreditación se ha erigido en el objetivo prioritario de las políticas nacionales de garantía de la calidad. Por una parte, se trata de reforzar la confianza entre los diplomas ofrecidos dentro del nuevo contexto. Para ello, los modelos tradicionales de garantía de la calidad heredados de la década de 1990 ya no parecen pertinentes, volveremos sobre este tema más adelante. La acreditación se puede entender, en este caso, como la respuesta a la variedad de modelos nacionales existentes. Por otra parte, la cons- 
trucción del EEES refuerza el proceso de competición entre las universidades a nivel nacional e internacional.

De forma similar, pero simbólicamente mucho más relevante, los acontecimientos actuales marcan la consagración de los objetivos sumativos de las políticas relativas a la garantía de la calidad. Este tipo de objetivos implican que las políticas persiguen de forma prioritaria los resultados de los diferentes centros universitarios y establecen, de forma más o menos directa, una relación entre estos resultados y los medios financieros puestos a disposición por las autoridades.

En cierta medida, esta consagración constituye el punto de llegada de un proceso más amplio dentro del cual las preocupaciones meramente formativas que prevalecían en las actividades de evaluación de la calidad han dejado paso de manera progresiva - pero inexorable - a preocupaciones relacionadas de forma directa con la eficiencia de los sistemas de educación superior y, de forma más precisa, de los establecimientos de educación superior. Los objetivos de mejora efectiva de las actividades pedagógicas y de gestión estaban en el centro de las primeras iniciativas desarrolladas en el ámbito europeo durante la primera mitad de la década de 1980 en los Países Bajos y Francia y, más tarde, en el resto del continente, incluyendo España (DES, 1985; Maassen, 1987; Mora, 1991; Vroeijenstijn y Acherman, 1990; Perellon, 2003). Sin entrar en los detalles, cabe notar que una de las razones que subyacen al cambio de perspectiva se encuentra en la consolidación de lo que un observador de los sistemas de educación superior ha llegado a llamar "estado evaluador» y la influencia cada vez más notable de teorías neoliberales dentro del ámbito de las políticas de educación superior (Neave, 1984, 1988). El énfasis puesto por las políticas de garantía de la calidad en las cuestiones de eficiencia han llevado a segundo plano las reflexiones y las preocupaciones relacionadas con los objetivos formativos de mejora.

La consolidación del «estado evaluador» nos lleva a subrayar un último punto de relevancia cuando se trata de analizar el nuevo contexto de las políticas de garantía de la calidad, a saber, su rol en las relaciones que mantienen las autoridades políticas con los sistemas de educación superior. Cabe notar que el desplazamiento de los objetivos de las políticas de calidad hacia la consagración de los objetivos sumativos corresponde a un refuerzo de los aspectos reguladores de los sistemas nacionales. De forma algo provocadora, es posible afirmar que lo que está aconteciendo en temas relacionados con las políticas de educación superior dentro del ámbito europeo —y seguramente mucho más allá de este espacio político - tiene muy poco que ver con preocupaciones centradas meramente en la «calidad» de la formación dispensada a los estudiantes y mucho más con la regulación de los sistemas nacionales de educación superior.

Un segundo elemento que cabe subrayar en relación con la emergencia de la acreditación, es que ésta última puede ser vista también como la respuesta a la gran diversidad que prevalece entre los diferentes países en cuestiones de políticas de garantía de la calidad (Vroeijenstijn, 2003). Esta variedad corresponde 
evidentemente a las trayectorias nacionales y a las configuraciones particulares de actores que, en cada caso, han aportado respuestas específicas en función del contexto social, político e institucional propio. La acreditación aparece entonces como una alternativa viable, tanto para la preservación de la diversidad nacional como para la necesaria convergencia entre los diferentes países, con el fin de conseguir el objetivo de armonización.

\section{Nuevas tendencias en el rol de los estudiantes}

Los cambios que hemos descrito anteriormente tienen un impacto significativo en el papel de los estudiantes en temas relacionados con la garantía de la calidad. A nuestro parecer, el impacto es de dos órdenes y traduce dos tendencias que, sin mantener una dependencia causal entre ellas, sí están relacionadas. El primer impacto puede traducirse en la presentación del estudiante como un "cliente», perspectiva que refuerza la tendencia hacia una mayor "comercialización» de la educación superior. El segundo impacto se relaciona más bien con las nuevas metodologías que están siendo desarrolladas con el objetivo de incluir de forma más directa a los estudiantes en los procesos de garantía de la calidad. Discutamos estas dos dimensiones.

La cuestión de la comercialización de la educación superior ha dado lugar a numerosos debates sobre las razones de su emergencia e influencia crecientes, sus características principales y sus consecuencias sobre las universidades (por ejemplo: Ginsburg y otros, 2003; Bok, 2003; Willmott 2003). Las presiones actuales sobre las universidades para que desarrollen su relación con las políticas relativas a la garantía de la calidad residen, según nosotros, en el desarrollo de un contexto económico y político, dentro del cual las universidades se constituyen como actores directamente involucrados en estrategias de mejora de sus condiciones de trabajo, mediante la captación de fuentes de financiación privadas. Esta tendencia se observa particularmente en el campo de la investigación (Geuna y Martin, 2003; Braddock y Neave, 2002) pero también afecta a las actividades de educación (Levy, 2003; Rolfe, 2002). Las transformaciones actuales del panorama de la educación superior en Europa constituyen, en este sentido, un contexto fértil dentro del cual la tendencia a una mayor atención a los elementos de funcionamiento de tipo mercado pueden seguir desarrollándose.

En el campo de la garantía de la calidad, un elemento principal reside en la utilización de los resultados de las evaluaciones que se realizan en los diversos programas o cursos (Vidal, 2000). Ya hemos notado el progresivo desbancamiento de objetivos formativos dentro de las evaluaciones en favor de objetivos sumativos, directamente relacionados con los resultados y la rendición de cuentas. En este contexto, los resultados de las evaluaciones ya no son prioritariamente en beneficio de los establecimientos, sino que se ponen mucho más al servicio de los actores exteriores, los stakeholders en inglés, entre los cuales los estudiantes y sus familias juegan un papel importante. Poniendo el acento sobre la dimensión sumativa, orientada hacia los resultados y su publicación, 
y situadas dentro de un marco reforzado de competición entre los establecimientos, las políticas de garantía de la calidad ponen cada vez más de manifiesto su tendencia a desarrollar estrategias relacionadas con principios de competencia. Por ejemplo, en los EEUU la competencia entre las universidades para atraer al mayor número de estudiantes posible no tiene comparación con ningún otro país. Existe una industria importante y rica, tremendamente rica, dedicada especialmente al reclutamiento de estudiantes. El contexto europeo actual otorga a esta temática una relevancia particular, en el sentido de que, a través de la construcción del EEES, se trata de asegurar la comparabilidad y el reconocimiento de los diplomas nacionales.

La cuestión del reconocimiento de los diplomas constituye un elemento muy importante dentro del debate sobre la comercialización de la educación superior y del papel que van a tener las nuevas formas de políticas de garantía de la calidad en este ámbito. En este sentido, y siguiendo lo que hemos notado más arriba, la consagración de la acreditación constituye un elemento fuerte del proceso de comercialización. Es más, constituye un elemento central de este proceso a través del «etiquetaje» que otorga a los diplomas reconocidos o a los establecimientos. Para realizar este objetivo, se requieren una serie de medidas, entre las cuales, la categorización de los resultados de las evaluaciones es la más significativa e importante.

En este sentido, desde hace ya varios años, se han generalizado las clasificaciones de los establecimientos de nuestras universidades, en función, parece ser, de su nivel de "calidad» respectivo. Desde que el semanario norteamericano US News and World Report publicó el primer ejercicio de este tipo en 1983, esta práctica, conocida en el mundo anglosajón por el término de ranking, ha adquirido una importancia creciente en un gran número de países (por ejemplo Bélanger y Davidson, 1997; Berghoff y otros, 2003; Dill y Soo, 2004; Eccles, 2002; Morse, 2001; Vaughn, 2002). Hoy en día, la influencia de los rankings anuales es tal que los establecimientos se deben casi por completo a la obligación de estar por encima de sus competidores directos, sean reales o percibidos como tales, en los diferentes criterios. Los sentimientos de los establecimientos de educación superior en relación con estos ejercicios varían en función de la posición particular de cada uno de ellos en los rankings.

No es éste el sitio para detallar los numerosos debates que han salpicado la cuestión de los rankings tanto desde el punto metodológico como de su pertinencia (Bowden, 2000; Escudero, 2003; Mora, 1993; Pike, 2004; Yorke, 1998). Lo que deseamos afirmar es que la construcción de los rankings y su puesta a disposición de los estudiantes como fuente de información privilegiada para la selección del establecimiento donde cursarán sus estudios, participa de forma directa de una visión construida alrededor de criterios de comercialización de la educación superior. Esta visión se basa en la idea según la cual hay que poner a disposición de los estudiantes y de sus familiares la mayor información posible sobre la "calidad» de las universidades. La relación entre esta visión de los esfuerzos en el tema de calidad y la idea de comercialización es fuerte. Supone que, una vez informado sobre el tipo de universidad y la cali- 
dad de sus programas, cada estudiante podrá elegir de manera informada. En el contexto de construcción del EEES, este elemento adquiere una dimensión crucial, puesto que la movilidad estudiantil que se persigue va acompañada de la necesidad evidente de permitirla y promoverla.

Una tal evolución transforma de forma radical y permanente el enfoque de las políticas de garantía de la calidad, en la medida en que nos encontramos delante de políticas cuyo énfasis se sitúa en los objetivos sumativos de rendición de cuentas, de regulación de los sistemas de educación superior y de puesta a disposición de la mayor información posible. Ejemplos significativos de estos casos pueden encontrarse en Australia, donde las informaciones recogidas sirven directamente para la elaboración de publicaciones de rankings para suministrar información a los estudiantes (DEYTA, 2000:14). De forma similar, el Reino Unido se ha caracterizado por un gran interés en imponer objetivos de rendición de cuentas. En este sentido, una de las misiones más significativas de la Quality Assurance Agency for Higher Education (QAA), el organismo responsable de asegurar la calidad de la educación superior, es, precisamente, la de diseminar información relacionada con la calidad de la educación (QAA, 2003; Randal, 2000). Esta información debe favorecer tanto a los estudiantes - en el momento de escoger el lugar donde cursar una carrera- como al mercado del trabajo y, de forma más general, a la sociedad.

El papel de los estudiantes en temas de calidad se transforma de otra manera. Éste se relaciona íntimamente con la cuestión de la rendición de cuentas y de la mayor comercialización de la educación superior que acabamos de poner de manifiesto como un elemento crucial en el desarrollo de los rankings, pero se distancia significativamente de estos procesos, en el sentido que los cambios afectan más directamente a las metodologías desarrolladas. La cuestión sigue relacionada con el tipo y la cantidad de información que se busca y con las formas de obtenerla. En el Reino Unido, estas preocupaciones han centrado los debates sobre la modificación de las estrategias de control y de garantía de la calidad de la educación universitaria tal como eran desarrolladas e implementadas desde finales de la década de 1990 (Brown, 2000; HEFCE, 1999; HEFCE y otros, 2001; Yorke, 1997). Los debates han puesto de manifiesto la necesidad de recomponer el rompecabezas de la calidad, centrándola en los estudiantes como fuente de información fundamental sobre el verdadero nivel de la formación que reciben (HEFCE, 2002; Brennan y Williams, 2004).

\section{Elementos de conclusión}

Las evoluciones discutidas en el presente artículo tienen un impacto directo sobre las políticas de garantía de la calidad formuladas e implementadas en el ámbito europeo. Según nuestro punto de vista, el hecho más significativo reside en el cambio en los objetivos de estas políticas, que han pasado a promocionar de manera directa los elementos sumativos en lugar de los elementos más formativos, es decir, a promover los elementos que ponen énfasis en la rendición de cuentas por parte de las universidades, así como una mayor «trans- 
parencia» $y$, de hecho, en la eficiencia de sus actividades, en lugar de preocupaciones más directamente relacionadas con el proceso de aprendizaje por parte de los estudiantes. Nos parece que esta evolución encuentra su punto de consagración en el reconocimiento de la acreditación como el objetivo prioritario de las políticas nacionales de garantía de la calidad. Esta evolución tiene hoy en día una imagen muy clara en la imposición de la acreditación como objetivo prioritario, y tal vez único, de las políticas de calidad. Este elemento es reciente y se inscribe dentro de las soluciones preconizadas para favorecer la comparabilidad de las prácticas nacionales en cuestiones relacionadas con la calidad de la educación superior. En este sentido, la emergencia de la acreditación dentro del ámbito europeo está íntimamente relacionada con las transformaciones acontecidas en el paisaje europeo de la educación superior, en particular con la construcción del espacio europeo de educación superior. Las transformaciones actuales corresponden, en cierta medida, a la convergencia de tendencias de larga duración que han marcado profundamente el paisaje de la educación superior durante las tres últimas décadas. Entre ellas, cabe subrayar el abandono del modelo tradicional de universidad y la emergencia y consolidación del «estado evaluador».

Esta evolución no se produce exenta de riesgo: las preocupaciones alrededor de la acreditación ponen fin a los esfuerzos realizados en favor de una evaluación formativa. Se pone también de relieve el riesgo importante de estandarización de los programas de estudios que conlleva la acreditación y la consecuente pérdida de variedad que sigue existiendo entre los establecimientos y, aún más importante, entre formas de abordar temáticas similares. En este sentido, y de forma provocadora, se puede afirmar que las evoluciones recientes en temas de políticas de garantía de la calidad, tienen cada vez menos que ver con la intención de promocionar verdaderamente la mejora de la pedagogía o de la gestión universitarias y mucho más con la intención de controlar y reglamentar los sistemas de educación superior.

Queda todavía por determinar en qué medida este nuevo contexto influye sobre las prácticas estudiantiles. Lo que es ya seguro, sin embargo, es que las transformaciones actuales tienden a incrementar el rol de los estudiantes en las orientaciones de la educación superior, particularmente en lo que se refiere a la presión que pueden ejercer sobre el tipo de formación que están dispuestos a recibir, las modalidades de transmisión de esta educación y, de forma más general, la calidad de esta formación.

\section{Bibliografía}

Altbach, P.G. (1999). «The Logic of Mass Higher Education». Tertiary Education and Management, 5(2): 107-124.

Ball, C. (1985). Fitness for Purpose. Essays in Higher Education. Edited by Dorma Urwin. Guildford. SRHE and NFER-Nelson.

BARnETT, R. (1992). Improving Higher Education. Total Quality Care. Buckingham: SRHE \& Open University Press. 
BÉLANGER, C.H.; DAVIDSON, R. (1997). «Ranking Canadian Universities: A Case of Controversy». Tertiary Education and Management, 3(1): 44-51.

BerGHOFF, S.; Federkeil, G.; GiebisCh, P.; HaChMeISTer, C.-D.; MÜller-BÖling, D. (2003). Das CHE Forschungsranking deutscher Universitäten. Gütersloh: Centrum für Hochschulentwicklung.

Berlin Communiqué (2003). Realising the European Higher Education Area. Communique of the Conference of Ministers responsible for Higher Education. Berlín, 19 de septiembre.

BoK, D. (2003). Universities in the Marketplace: The Commercialization of Higher Education. New Jersey: Princeton University Press.

Bologna DeClaration (1999). The European Higher Education Area. Jointdeclaration of the European Ministers of Education. Bolonia, 18 y 19 de junio.

Bowden, R. (2000). «Fantasy Higher Education: University and College League Tables». Quality in Higher Education, 6(1): 41-60.

Bowden J.; Marton, F. (1998). The University of Learning. Beyond Quality and Competence in Higher Education. Londres: Kogan Page.

BRADDOCK, R.; NEAVE, G. (2002). «Research management in higher education: overview and conclusion of a debate». Higher Education Policy, 15(3): 313-330.

Brennan, J.; GoedegebuUre, L.C.J.; SHAh, T.; Westerheijden, D.F.; Weusthof, P.J.M. (1992). Towards a Methodology for Comparative Quality Assessment in European Higher Education. Londres: CNAA, CHEPS, HIS.

BrenNan, J.; Williams, R. (2004). Collecting and using student feedback. A guide to good practice. Londres: Center for Higher Education Research and Information.

BROwN, R. (2000). «The New UK Quality Framework». Higher Education Quarterly, 54(4): 232-242.

CEC (1995). A Comparative Analysis of Initiatives of Quality Assurance and Assessment of Higher Education in Europe. Bruselas: Commission of the European Communities.

CoEC (2003). Communication from the Commission. The role of the universities in the Europe of knoweldge. Bruselas: Commission of the European Communities.

CrowtHeR-HunTeR, L. (1983). «Policy-Making and Accountability in Higher Education». En SHATTOCK, M. (ed.). The Structure and Governance of Higher Education. Guildford: Society for Research into Higher Education, p. 46-67.

DEPARTMENT OF EDUCATION AND SCIENCE (DES) (1985), The Development of Higher Education into the 1990s. Londres: HMSO.

DETYA (2000). The Australian Higher Education Quality Assurance Framework. Canberra: Department for Education, Training and Youth Affairs.

Dill, D.D.; SoO, M. (2004). «Is There a Global Definition of Academic Quality? A Cross-National Analysis of University Ranking Systems». Presentación en la International Network of Quality Assurance Agencies in Higher Education, Dublín, 17 de abril.

DunN, D.D. (2003). «Accountability, Democratic Theory, and Higher Education». Educational Policy, 17(1): 60-79.

EC (2003). Berlin Conference of European Higher Education Ministers "Realising the European Higher Education Area». Contribution of the European Commission. Bruselas: European Commission.

ECCLES, C. (2002). «The Use of University Rankings in the United Kingdom». Higher Education in Europe, XXVII(4): 423-432.

ESCUDERO, T. (2003). «El fraude conceptual de los rankings de universidades». El Pais, 13 de enero. 
Geuna, A.; Martin, B. (2003). «University Research Evaluation and Funding. An International Comparison». Minerva, 41(4): 277-304.

Ginsburg, M.; EsPInOZA, O.; Popa, S.; Terano, M. (2003). «Privatisation, Domestic Marketisation and International Commercialisation of Higher Education: vulnerabilities and opportunities for Chile and Romania within the framework of WTO/GATS». Globalisation, Societies and Education, 1(3): 413-445.

Green, D. (1994). «What is Quality in Higher Education ? Concepts, Policy and Practice». En GREEN, D. (ed.). What is Quality in Higher Education? Buckingham: SRHE \& The Open University Press, p. 3-20.

HAAKSTAD, J. (2001). «Accreditation: The New Quality Assurance Formula? Some Reflections as Norway is about to Reform its Quality Assurance System». Quality in Higher Education, 7(1): 77-82.

HACKL, E. (2001). Towards a European Area of Higher Education: Change and Convergence in European Higher Education. Florencia: Robert Schuman Centre for Advanced Studies.

Harvey, L.; Green, D. (1993). «Defining Quality». Assessment and Evaluation in Higher Education, 18(1): 9-34.

Haug, G. (2003). «Quality Assurance/Accreditation in the Emerging European Higher Education Area: a possible scenario for the future». European Journal of Education, 38(3): 229-240.

HEFCE (1999). Providing Public Information on the Quality and Standards of Higher Education Courses. Bristol: Higher Education Funding Council for England.

- (2002). Information on quality and standards in higher education. Final report of the Task Group. Bristol: Higher Education Funding for England.

HEFCE; UUK; SCOP (2001). Quality Assurance in Higher Education: Delivering Lightness of Touch. Londres: Higher Education Funding Council for England.

Henkel, M. (2002). «Academic Identity in Transformation? The Case of the United Kingdom». Higher Education Management and Policy, 14(3): 137-147.

Kivinen, O.; NuRMI, J. (2003). «Unifying Higher Education for Different Kinds of Europeans. Higher Education and Work: a comparison of ten countries». Comparative Education, 39(1): 83-103.

KNIGHT, J. (2002). «Trade Talk: An Analysis of the Impact of Trade Liberalization and the General Agreement on Trade in Services on Higher Education». Journal of Studies in International Education, 6(3): 209-229.

KoGAN, M. (1986). Education accountability : an analytic overview. Londres: Hutchinson Educational.

LeVY, D.C. (2003). Expanding Higher Education Capacity Through Private Growth. Contributions and Challenges. Londres: Observatory on Borderless Higher Education. MAASSEN, P.A.M. (1987). «Quality Control in Dutch Higher Education: Internal versus External Evaluation». European Journal of Education, 22(2): 161-170.

MARTENS, K.; BALZER, C. (2004). «Comparing Governance of International Organisations - The EU, the OECD and Educational Policy». Contribución a la European Consortium for Political Research Joint Sessions. Uppsala, del 13 al 18 de abril.

Middlehurst, R. (1992). "Quality: An Organising Principle for Higher Education?». Higher Education Quarterly, 46(1): 20-38.

- (2002). "The Development of World Borderless Higher Education: Markets, Providers, Quality Assurance and Qualifications». Contribución a la First Global Forum on International Quality Assurance, Accreditation and the Recognition of Qualifications in Higher Education. París, 17 y 18 octubre. 
Miguel, M. de y otros (1994). Evaluación para la calidad de los institutos de educación secundaria. Madrid : Editorial Escuela Española.

MorA, J.-G. (1991). Calidad y Rendimiento en las Instituciones Universitarias. Madrid: Consejo de Universidades.

- (1993). «Evaluación de la Calidad de la Universidad». Universidades, III: 5-19. MorSE, R. (2001). «U.S. News \&World Report's College Rankings». Paper presentation at Association for Institutional Research Annual Forum. Long Beach, California, del 3 al 6 de junio.

MuÑOZ-RePISO, M. (1996). «La calidad como meta». Cuadernos de Pedagogía, 246, 52-57. Neave, G. (1984). «On The Road to Silicon Valley? The Changing Relationships Between Higher Education and Government in Western Europe». European Journal of Education, 19(2): 111-129.

- (1988). «On the Cultivation of Quality, Efficiency and Enterprise. An Overview of Recent Trends in Higher Education in Western Europe». European Journal of Educaion, 23(1/2): 7-23.

- (2003). «The Bologna Declaration: Some of the Historic Dilemmas Posed by the Reconstruction of the Community in Europe's Systems of Higher Education». Educational Policy, 17(1): 141-164.

Neave, G.; Van Vught, F. A. (eds.) (1991). Prometheus Bound. The Changing Relationship Between Government and Higher Education in Western Europe. Oxford (UK)/Nueva York: Pergamon Press.

Perellon, J.-F. (2003). La qualité dans l'enseignement supérieur. Reconnaissance des filières d'études en Suisse et en Europe: Analyse d'une révolution. Lausana: Presses Polytechniques Universitaires Romandes.

PIKE, G.R. (2004). «Measureing Quality: A Comparison of U.S. News Rankings and NSSE Benchmarks». Research in Higher Education, 45(2): 193-208.

PolanyI, M. (1966). The Tacit Dimension. Londres: Routledge \& Kegan Paul.

QAA (2003). Learning from Subject Review 1993-2001. Sharing good practice. Gloucester: Quality Assurance Agency.

Randall, J. (2002). "Quality Assurance: Meeting the Needs of the User». Higher Education Quarterly, 56(2): 188-203.

Rolfe, H. (2002). «Students' Demands and Expectations in an Age of Reduced Financial Support: the perspectives of lecturers in four English universities». Journal of Higher Education Policy and Management, 24(2): 171-182.

SCOTT, P. (1995). The Meanings of Mass Higher Education. Buckingham: SRHE \& Open University Press.

TAM, M. (2001). «Measuring Quality and Performance in Higher Education». Quality in Higher Education, 7(1): 47-54.

Thune, C.; Staropoli, A. (1997). «The European Pilot Project for Evaluating Quality in Higher Education». En Brennan, J.; VRIES, P.D.; Williams, R. (eds.). Standards and Quality in Higher Education, 198-204. Londres: Jessica Kingsley.

Trow, M. (1974). «Problems in the Transition from Elite to Mass Higher Education». En OECD (ed.). Policies for Higher Education. París: OECD, p. 51-101.

VAN DAMmE, D. (2002). "Trends and Models in International Quality Assurance in Higher Education in Relation to Trade in Education». Higher Education Management and Policy, 14(3): 93-136.

VAN DER Wende, M. (2000). «The Bologna Declaration: Enhancing the Transparency and Competitiveness of European Higher Education». Higher Education in Europe, XXV(3): 305-310. 
Vaughn, J. (2002). «Accreditation, Commercial Rankings, and New Approaches to Assessing the Quality of University Research and Education Programmes in the United Status». Higher Education in Europe, XXVII(4): 433-441.

VIDAL, J. (2000). «Implementing Improvement Actions at Universities». Contribucion What Use Can be Made of the Outcomes of Evaluations? Barcelona, 17-18 de noviembre.

VRoeijenstijn, T.A. (1992). «External Quality Assessment, Servant of Two Masters? The Netherlands University Perspective». En CrafT, A. (ed.). Quality Assurance in Higher Education. Proceedings of an International Conference. Hong Kong 1991. Londres: Falmer Press, p. 109-131.

- (2003). Similarities and Differences in Accreditation. Looking for a Common Framework. The Hague: The Netherlands Accreditation Organisation.

VROEIJENSTIJN, T.A.; ACHERMAN, J.A. (1990). «Control Oriented Versus Improvement Oriented Quality Assessment». En GoedegebuUre, L.C.J.; MaAssen, P.A.M.; Westerheijden, D.F. (eds.). Peer Review and Performance Indicators: Quality Assessment in British and Dutch Higher Education. Utrecht: Lemma, p. 81-101.

WeSTERHEIJDEN, D.F. (2003a). «Movements towards a European Dimension in Quality Assurance and Accreditation». En WesterheijDen, D.F.; LeEGWATER, M. (eds.). Working on the European Dimension of Quality. Report of the conference on quality assurance in higher education as part of the Bologna process. Zoetemeer: Ministry of Education, Culture and Sciences, p. 16-41.

- (2003b). «Accreditation in Western Europe: Adequate Reactions to Bologna Declaration and the General Agreement on Trade in Services?». Journal of Studies in International Education, 7(3): 277-302.

Westerheijden, D.F.; Van der Wende, M. (2001), «Who says B also has to say A? From Bologna to Accreditation: Design Requirements for Quality Assurance in Europe». Contribución a la Conferencia de la International Network of Quality Assurance Agencies in Higher Education. Bengalore, del 19 al 22 de marzo.

Willmott, H. (2003). "Commercialising Higher Education in the UK: the state, industry and peer review». Studies in Higher Education, 28(2): 129-141.

Yorke, M. (1997). «This Way QA?». Quality Assurance in Education, 5(2): 97-100.

- (1998). "The Times' "League Table" of Universities 1997. A Statistical Appraisal». Quality Assurance in Education, 6(1): 58-60.

Zgaga, P. (2003). Bologna Process: Between Prague and Berlin. Berlín: Report to the Ministers of Education of the signatory countries. 